\title{
Empatía en el contexto romántico: diseño y validación de una medida*
}

\section{Empathy in the Romantic Context: Design and Validation of a Measure}

Recibido: 29 de diciembre de 2012 | Aceptado: 29 de agosto de 2015

\author{
Rozzana SánChez Aragón ** \\ Magali Martínez PÉREZ **** \\ Universidad Nacional Autónoma de México, \\ Ciudad de México, México
}

doi:10.11144/Javeriana.upsy15-1.ecrd

Para citar este artículo: Sánchez-Aragón, R., \& Martínez-Pérez, M. (2016). Empatía en el contexto romántico: diseño y validación de una medida. Universitas Psychologica, 15(1), 19-28. http://dx.doi. org/10.11144/Javeriana.upsy15-1.ecrd

* Artículo de investigación. Proyectos financiados por la DGAPA-UNAM PAPITT IN 302811 e IN 301814.

*** Profesora Titular de Tiempo Completo en la Facultad de Psicología de la Universidad Nacional Autónoma de México desde hace 20 años. Miembro del Sistema Nacional de Investigadores, Nivel II. Correo electrónico: rozzara@unam.mx

**** Estudiante de Doctorado. Correo electrónico: magy0313@yahoo.com.mx

\section{RESUMEN}

La empatía en su naturaleza involucra una serie de nociones (Batson, 2009) cuyos énfasis van de menos a más en cuanto a dos elementos psicológicos fundamentales: la cognición y la emoción que se vinculan en uno u otro sentido, es decir, en un carácter de comprensión intelectual o imaginativa de la condición del otro sin experimentar realmente los sentimientos de esa persona (e.g., Hogan, 1969) o como una reacción emocional o simpatía en respuesta a los sentimientos o experiencias de otros (e.g., Mehrabian \& Epstein, 1972). Dado el carácter inherentemente interaccional de la empatía, el contexto de la relación de pareja se vuelve sumamente interesante de explorar en virtud de ella, ya que precisamente este vínculo, incluye matices de emocionalidad y cognición muy particulares que favorecen el despliegue de dicha variable que implicará la integración de sus miembros en una lectura cognitivo-emocional característica y por tanto, determinará el grado de intimidad e inmediatez alcanzado por ellos. Ante esto, el propósito de este estudio fue diseñar y validar una medida de empatía hacia la pareja en una muestra no probabilística propositiva de 467 adultos de la Ciudad de México que contaban con una relación romántica. Los resultados muestran la obtención de cuatro factores clave en el entendimiento de la variable como lo son: toma de perspectiva, empatía cognoscitiva de las emociones, perturbación propia y compasión empática. Dichos factores mostraron validez de constructo y coeficientes alpha de Cronbach moderados y en su conjunto representa una contribución conceptual, metodológica y cultural al estudio de la variable en el contexto romántico.

Palabras clave

empatía; pareja; cognición; emociones; medición

\section{A B S T R A C T}

Empathy involves in its nature a series of other notions (Batson, 2009) whose highlights comprise two psychological elements in different degrees: cognition and emotion, which are linked each other. In one hand, empathy can be a kind of intellectual or imaginative comprehension about other without really experience the feeling of that person (i.e. Hogan, 1969), on the other hand, empathy is an emotional reaction or sympathy in response to the feelings or experiences of others (i.e. Mehrabian \& Epstein, 1972). Due to its inherent interactional character of empathy, the couple relationship context 
becomes very interesting to explore; also because it includes a particular emotional and cognitive shades that favor the display of that variable which involve the integration of its members in a characteristic cognitive-emotional reading and thus determine the degree of intimacy and immediacy achieved by them. At this, the purpose of this research was to design and validate an empathy toward the partner measure in an intentional non probabilistic sample of 467 adults from Mexico City whose had a romantic relationship at the moment of the study. Findings show the extraction of four key factors in the understanding of empathy: perspective taking, cognitive empathy of emotions, self-disturbance and empathetic compassion. Those factors showed construct validity and moderate Cronbach reliability coefficients and they represent a conceptual, methodological and cultural contribution to the variable in the romantic context.

Keywords

empathy; partner; cognition; emotion; measure

Las complejidades de la vida humana frecuentemente necesitan poseer cierta sensibilidad hacia la conducta de otros. De ahí, que la atención hacia las experiencias de otros cuando enfrentan algún daño físico y/o dolor es un elemento de gran relevancia tanto para la sobrevivencia de una persona como la de un grupo. Por ello, conocer los sentimientos, pensamientos y reacciones conductuales de otros en el contexto de lo que les sucede, puede ser vital si quien observa se protege a sí mismo o brinda cuidado a la otra persona. Aunque es reconocido que los precursores de la capacidad de empatizar están biológica y conductualmente presentes y conservadas en especies no humanas (e.g., Preston \& de Waal, 2002), las ciencias sociales y de la conducta han generado distintas acepciones que expresan distintas aristas que definen y delimitan al constructo (Batson, 2009).

En 1909 Lipps utilizó por vez primera en la literatura psicológica el término de empatía (sentimiento de unión con), y propuso que a través de un proceso de imitación motora, un observador siente -al presenciar la experiencia emocional de un actor-, la misma emoción que el actor. Esta misma visión de la empatía fue compartida por Gordon (1995) y definida como empatía facial o mimetismo motor (Hoffman, 2000). No obstante, Khöler (1947) planteó que la empatía implica entender o conocer, pero no necesariamente compartir las emociones de otros; postura que fue apoyada por autores como
Preston y de Waal (2002) quienes llamaron a este conocimiento empatía cognoscitiva. Otras definiciones de empatía se centraron en la idea de que esta es más una reacción emocional o simpatía, en respuesta a los sentimientos o experiencias de otros, señalando que implica una "mayor receptividad a la experiencia emocional de otro" (Mehrabian \& Epstein, 1972; p. 526) y definida de acuerdo con Darwall (1998) y Preston \& de Waal (1998) como piedad o compasión empática. En tanto, autores como Hogan (1969) describieron la empatía como "la comprensión intelectual o imaginativa de la condición del otro sin experimentar realmente los sentimientos de esa persona" (p. 308). Asimismo Stotland (1969) le llamó toma de perspectiva o imaginar al otro (Batson, 1991).

Como una forma de integrar las aproximaciones previas, Nickerson, Butler y Carlin (2011) la palabra empatía expresa la noción de compartir en forma vicaria un sentimiento, empatizar con otro es imaginarse a sí mismo en la situación de otra persona y vivenciar -en cierto grado- las emociones que el otro está experimentando, hallazgo sustentado ampliamente por estudiosos como Bernhardt y Singer (2012). Y lo complementa Hatfield, Rapson y Le (2009), que la definen como un consenso en la literatura clínica, y plantean que la verdadera empatía requiere tres habilidades distintas: la habilidad de compartir las experiencias afectivas de otra persona (Singer \& Lamm, 2009), la habilidad cognoscitiva de intuir lo que la otra persona está sintiendo y una intención "socialmente benéfica" a responder compasivamente al estrés de esa persona (Decety \& Jackson, 2004).

Este desacuerdo conceptual, sentó las bases para que en la conceptualización del término empatía, se reconocieran dos amplias categorías de respuesta: una más visceral, irreflexiva, instintiva o emocional -como plantean Mehrabian y Epstein (1972) - ; y una más cognoscitiva o intelectual -como indican Khöler (1947) y Hogan (1969). De esta manera, diversos autores (e.g., Díaz-Loving, González-Varela, La Rosa, \& Nina-Estrella, 1985; Eisenberg, 2000; Feshbach, N. D. \& Feshbach, S., 2011; Hojat et al., 2002; Nickerson et al., 2011) sugieren que sería un error considerar exclusivamente lo emocional o 
lo cognoscitivo al definir el constructo. Bajo esta lógica, pueden observarse definiciones de empatía que integran ambos elementos.

Por ejemplo, Díaz-Loving et al. (1985) señalan que es a través de la empatía que un individuo se hace consciente de los sentimientos, pensamientos e intenciones de otro (cognición), lo cual puede llevarlo a una respuesta afectiva vicaria (emoción). De manera similar, Eisenberg (2000) define a la empatía como una respuesta emocional que procede de la comprensión del estado o situación de otra persona y es similar a lo que la otra persona está sintiendo. Más recientemente, Hojat et al., (2002) indicaron que la empatía podía ser descrita como un concepto en el que participan el dominio cognoscitivo y el emocional.

Pese a que se logró un consenso con respecto a la integración de ambos dominios, otro dilema está presente en el estudio de la empatía, y este tiene que ver con la multidimensionalidad del mismo (cf. Davis, 1983; Nedelsticher-Mitrani, Díaz-Loving, \& Nina-Estrella, 1983). Esto quiere decir, que aún en las categorías básicas de cognición y emoción, existen subdivisiones y es Batson (2009) quien reconoce al menos ocho usos distintos del término. Dada la controversia entre las dimensiones que integran el constructo de empatía, el interés en su estudio ha ido en aumento; lo que ha generado una serie de distinciones teóricas y operacionales sobre las áreas que la conforman.

\section{Midiendo la empatía}

Los instrumentos a través de los cuales no va acentuada se ha intentado operacionalizar a la empatía, reflejan ya sea la distinción fundamental entre cognición y emoción, o una visión integradora de dichos elementos. Por ejemplo, Hogan (1969) dentro del enfoque cognitivo desarrolló la Hogan Empathy Scale (EM por sus siglas en inglés), que fue la más utilizada durante años dado que se compone de 64 reactivos y su tiempo de administración es breve; además de que posee adecuadas garantías psicométricas.

Por su parte, Mehrabian y Epstein (1972) desarrollaron el Questionnaire Measure of Emotional
Empathy (QMEE, por sus siglas en inglés) para medir la empatía, en su lado emocional. Dicho cuestionario evalúa una amplia variedad de reacciones emocionales a un número extenso de estímulos a través de siete subescalas altamente correlacionadas entre sí, suponiendo la unidimensionalidad de esta forma de empatía. No obstante, en una investigación con mexicanos, Nedelsticher-Mitrani et al., (1983) encontraron que al analizar factorialmente al QMEE surgían otras dimensiones diferentes a las observadas en la escala original. Y años más tarde Fernández-Pinto, López-Pérez, y Márquez (2008) señalarían que algunas de sus subescalas eran redundantes (e.g., Tendencia a la compasión y Tendencia a conmoverse por los estados negativos de los otros).

Dentro de las escalas con una visión integradora de la empatía se encuentra el Índice de Reactividad Interpersonal (IRI) de Davis (1983). El IRI permite evaluar la disposición empática a través de cuatro factores (dos cognoscitivos y dos emocionales). Dentro de los primeros se encuentran la Toma de perspectiva (i.e., la habilidad para comprender el punto de vista de la otra persona) y la Fantasía (i.e., capacidad imaginativa del sujeto para ponerse en situaciones ficticias). Los factores que corresponden al área emocional son la Preocupación empática (i.e., sentimientos de compasión, preocupación y cariño por otros) y el Malestar personal (i.e., sentimientos de ansiedad y malestar que el sujeto manifiesta al observar las experiencias negativas de los demás).

Pérez-Albéniz, De Paúl, Etxeberría, Montes y Torres (2003) adaptaron al español el IRI empleando la técnica de traducción-retraducción y lo validaron en una muestra de estudiantes de la Universidad del País Vasco. Los resultados del análisis factorial mostraron cuatro dimensiones muy similares en términos estructurales a las del instrumento original, a saber: Toma de perspectiva, Fantasía, Preocupación empática y Perturbación personal

Retomando la propuesta conceptual de empatía integral de Davis (1983), Díaz-Loving, AndradePalos y Nadelsticher-Mitrani (1986) decidieron construir la Escala de Apreciación y Sensibilidad Emocional (EASE) para una muestra de la Cd. de México. Los factores obtenidos fueron: Compasión 
empática (e.g., sentimientos de simpatía y compasión hacia otros), Perturbación propia (e.g., sentimientos negativos en el observador provocados por el sufrimiento de otros), Empatía cognoscitiva (e.g., percepción de los estados anímicos y emocionales de otros) e Indiferencia-Tranquilidad (e.g., indiferencia y tranquilidad ante los problemas de otros); todos con coeficientes de confiabilidad moderados a altos (coeficientes de 0.77 a 0.89).

Además de los instrumentos hasta aquí descritos se han desarrollado otros intentos para medir a la empatía en contextos específicos, por ejemplo, en la relación médico-paciente. Uno de las escalas más empleadas es la Escala de Empatía Médica de Jefferson (EEMJ, Hojat et al., 2002) que evalúa la empatía del personal de enfermería y médicos a través de tres factores: Toma de perspectiva (e.g., trato de entender lo que está pasando en la mente de mis pacientes, prestando atención a sus señales no verbales y el lenguaje corporal), Cuidado compasivo (p.ej., considero que preguntar a los pacientes sobre lo que está sucediendo en sus vidas un factor de importancia en la comprensión de sus molestias físicas) y Pensar como el paciente (e.g., es difícil para mí ver las cosas desde la perspectiva de mis pacientes).

Esta propuesta de medición sugiere que resulta de vital importancia contar con instrumentos que permitan medir la empatía en contextos relacionales específicos, sobre todo aquellos en los que las emociones constituyen un elemento clave. En consecuencia, medir la empatía en el contexto romántico, que es vislumbrado como el campo más fértil para la experiencia emocional (Carlson \& Hatfield, 1992), es fundamental; ya que dependiendo de cómo los miembros de la diada manejan dichos estados afectivos (sobre todo los negativos), resultará en el éxito o fracaso de la relación (Perlman, 2000).

\section{La empatía en la relación romántica}

En el contexto romántico se ha encontrado que las habilidades empáticas, tales como la capacidad para entender a la pareja y ponerse en su lugar, favorecen la interacción y llevan a percibir al otro como un ser individual y único de una manera más realista, sin adjudicarle características basadas en expecta- tivas propias (Carrillo, 1999). No obstante, se ha encontrado que cuando han transcurrido más años de casados existe una tendencia a entender mejor las emociones del otro y no solo eso, sino que existe también una percepción de que el otro entiende mejor a su pareja (Carrillo, 1999).

Más recientemente, Aguilera (2009) encuentra que las personas con mayor comprensión empática (e.g., mayor capacidad para estar consciente de lo que en ese momento le ocurre a la pareja internamente) emplean un estilo de negociación integrativo para solucionar sus conflictos; es decir, los solucionan a través de la propuesta de alternativas en las que puedan integrarse tanto sus propios intereses como los de su pareja.

Por otro lado, la falta de empatía entre los miembros de la pareja, facilita la violencia en las parejas jóvenes y adultas (González-Ortega, Echeburúa, \& Corral, 2008). De ahí que, algunas de las intervenciones encaminadas a la prevención del maltrato psicológico en la pareja señalen la necesidad de establecer relaciones de pareja basadas en la empatía -entre otros aspectos- (Blázquez-Alonso, MorenoManso, \& García-Baamonde Sánchez, 2009).

Ahora bien, como puede observarse la investigación sobre la empatía en el contexto de la pareja romántica es escasa, aunado a ello, las medidas aplicadas en los estudios son generales y no evalúan la empatía en el marco del contexto relacional de pareja-aunque se han reconocido sus implicaciones diádicas-; además de que, únicamente se cuenta con una sola medida de empatía desarrollada en México, la EASE (Díaz-Loving et al., 1986), y sigue habiendo controversia sobre las dimensiones que integran el constructo. Es por ello, que el propósito del presente estudio fue operacionalizar el concepto de empatía hacia la pareja romántica, con el fin de obtener un instrumento válido y confiable para su medición.

\section{Método}

\section{Participantes}

Se trabajó con una muestra no probabilística propositiva (Kerlinger \& Lee, 2002) de 467 habitantes de la Ciudad de México, de los cuales 224 eran hom- 
bres y 237 mujeres, cuyas edades oscilaron entre los 17 y 80 años $(M=29.33, D E=11.61)$. De la muestra total, 199 se declararon solteros, 173 casados, 91 señalaron vivir en unión libre y 4 no respondieron cuál era su estado civil. En cuanto a la escolaridad, 168 participantes tenían estudios de preparatoria, 241 de licenciatura y 37 de posgrado (26 no respondieron). Todos los participantes informaron tener una relación de pareja al momento de participar en el estudio desde hacía al menos 3 meses y hasta 62 años $(M=7.39$ años, $D E=8.54)$.

\section{Instrumento}

Se diseñó la Escala de Empatía Romántica (EER) tomando en consideración tanto las dimensiones de la empatía propuestas en el IRI (Davis, 1983), la EASE (Díaz-Loving et al., 1986) y la EEMJ (Hojat et al. 2002); como algunas definiciones de empatía (e.g., Hogan, 1969; Mehrabian \& Epstein, 1972). Una vez generada una base amplia de reactivos apropiados para el contexto de la relación romántica se logró una escala con 71 afirmaciones que muestreaban cinco dimensiones de la empatía: toma de perspectiva (13), cuidado empático (13), compasión empática (15), preocupación propia (17) y empatía cognoscitiva (13). El formato de respuesta fue tipo Likert con cinco opciones de respuesta indicando grados de acuerdo.

\section{Procedimiento}

Los cuestionarios fueron aplicados por cuatro psicólogos que acudieron a lugares en donde se podían encontrar personas con una relación de pareja actual (plazas comerciales, casas, escuelas, oficinas, etc.), para que contestaran de forma voluntaria, anónima y confidencial la escala en cuestión. Durante la aplicación se dio respuesta inmediata a las dudas, asimismo se pusieron a disposición de los participantes sus resultados personales.

\section{Análisis de datos}

Con el fin de obtener una medida válida y confiable se realizaron los siguientes análisis estadísticos:
1) análisis de frecuencias reactivo por reactivo, 2) se aplicó una $t$ de Student para muestras independientes (reactivo por reactivo), 3) con los reactivos que aprobaron los análisis anteriores se realizó un análisis Alpha de Cronbach, para evaluar la consistencia interna de la escala, y 4) varios análisis de inter-correlaciones de los reactivos para determinar el tipo de rotación del análisis factorial.

\section{Resultados}

Ya ejecutados cada uno de los análisis estadísticos encaminados a identificar los reactivos con mayor poder discriminativo, se observó que 39 de los 71 ítems ${ }^{1}$ diseñados cumplieron con los criterios establecidos por Reyes-Lagunes y García y Barragán (2008), es decir, se encontraron dentro del rango de sesgo de -0.5 y +0.5 pues se consideró que la variable se distribuyó de una manera normal y que los cuartiles extremos de cada reactivo presentaron diferencias estadísticamente significativas. Acto seguido, se analizaron los reactivos por medio de un análisis factorial de componentes principales con rotación ortogonal (tipo varimax) debido a que al hacer un análisis de intercorrelaciones con los reactivos que discriminaron, estos mostraron coeficientes medianos, y se seleccionaron solo aquellos que registraron cargas factoriales iguales o superiores a 0.40. El análisis factorial arrojó un índice $\mathrm{KMO}=0.924$ y la Prueba de Esfericidad de Bartlett $=6155.060, \mathrm{gl}=780, p=0$ ) y Alpha de Cronbach total de 0.909. Además de indicar la existencia de 4 factores con valores superiores a 1 que explicaron el $40.35 \%$ de la varianza y con coeficientes de confiabilidad Alpha de Cronbach de moderados a moderados altos (ver Tabla 1).

\section{Discusión}

El estudio y entendimiento de la empatía en el contexto relacional romántico se basa en que "ser capaz de entender los estados mentales y afectivos de otra persona, le permite al individuo no solo

1 Estos reactivos tenían un coeficiente Alpha de Cronbach de 0.903 
TABLA 1.

Análisis factorial de la Escala de Empatía Romántica

Toma de perspectiva

A veces intento entender mejor a mi pareja imaginando cómo ve las cosas desde su perspectiva

$\alpha=0.85$

En un desacuerdo con mi pareja, trato de ver las cosas desde su punto de vista

0.625

Trato de pensar como mi pareja con el fin de ser más sensible a lo que le pasa

0.568

Creo que todas las cuestiones se pueden ver desde dos perspectivas e intento considerar ambas

0.567

Trato de imaginarme en los zapatos de mi pareja cuando así se requiere

0.533

Percibo sencillamente los cambios emocionales de mi pareja

0.531

0.520

Trato de entender lo que está pasando en la mente de mi pareja, prestando atención a sus señales no verbales $\quad 0.479$

y lenguaje corporal

Fácilmente me doy cuenta del estado de ánimo de mi pareja

0.469

Antes de criticar a mi pareja, intento imaginar cómo me sentiría si estuviera en su lugar

0.468

Suelo entender muy bien cómo hacer sentir bien a mi pareja

0.450

Soy muy perceptivo(a) de lo que pasa a mi alrededor

0.453

Creo que es fundamental atender a las necesidades emocionales de mi pareja

0.429

Creo que la empatía es un ingrediente importante en la relación con mi pareja

0.424

Entiendo fácilmente los sentimientos de mi pareja

0.422

Me siento muy contento(a) con las experiencias emocionales de mi pareja

0.412

Media $=3.76$ DS $=0.5917$

Empatía cognoscitiva de las emociones

$\alpha=0.82$

Sé cuándo mi pareja está enojada

0.610

Me doy cuenta cuando mi pareja "anda sentimental"

0.555

Percibo cuando alguien no le simpatiza a mi pareja

0.527

Cuando veo que se aprovechan de mi pareja, siento necesidad de protegerle $\quad 0.461$

Siento cuando algo anda mal con mi pareja aunque ella no me diga $\quad 0.459$

$\begin{array}{ll}\text { Distingo claramente cuando mi pareja se siente triste } & 0.452\end{array}$

Me doy cuenta cuando hago algo que le molesta a mi pareja $\quad 0.450$

Yo hago lo que sea necesario para que mi pareja sea feliz $\quad 0.443$

$\begin{array}{ll}\text { Me preocupo por mi pareja } & 0.429\end{array}$

Creo que preguntarle a mi pareja sobre lo que le pasa es básico en nuestra relación $\quad 0.414$

Media $=4.08$ DS $=0.5756$

Perturbación propia

$\alpha=0.70$

Me pone nervioso(a) si mi pareja se lastima

0.816

Me angustia tener una situación de emergencia donde mi pareja esté involucrada $\quad 0.594$

$\begin{array}{ll}\text { Yo sufro si veo que mi pareja no está feliz } & 0.583\end{array}$

$\begin{array}{ll}\text { Me inquieta ver a mi pareja lastimada } & 0.449\end{array}$

$\begin{array}{ll}\text { Me da pesar el ver sufrir a mi pareja } & 0.415\end{array}$

Media $=3.80 \mathrm{DS}=0.727$

Compasión empática $\alpha=0.78$

No soy indiferente a los problemas de mi pareja

0.710

$\begin{array}{ll}\text { Trato de prestar atención a las emociones de mi pareja } & 0.656\end{array}$

Soy el primero en enterarme sobre cómo se siente mi pareja $\quad 0.620$

$\begin{array}{ll}\text { Es relevante para mí el estado emocional de mi pareja } & 0.588\end{array}$

$\begin{array}{ll}\text { Me siento mal si veo llorar a mi pareja } & 0.479\end{array}$

En general tomo en cuenta los sentimientos de mi pareja $\quad 0.453$

$\begin{array}{ll}\text { Trato de ayudar a mi pareja cuando más lo necesita } & 0.434\end{array}$

Mi pareja se siente mejor cuando entiendo cómo se siente $\quad 0.402$

$\begin{array}{ll}\text { Me hace sentir bien ver la alegría de mi pareja } & 0.402\end{array}$

Media $=4.05$ DS $=0.6620$

Fuente: elaboración propia 
comunicarse e interactuar en formas efectivas y placenteras sino también predecir sus acciones, intenciones y sentimientos" (Singer \& Lamm, 2009; p. 81); esto se maximiza en el contexto de la pareja ya que la empatía evoluciona a la par de la comunicación entre sus miembros (Guerrero, Andersen, \& Afifi, 2011) y la intimidad (Laurenceau \& Kleinman, 2006) constituyendo con ello una conexión y apego que da cabida a su naturaleza comunal y diádica no parecida a ninguna otra (Aron, Aron, \& Smollan, 1992).

Así, el propósito del presente estudio fue operacionalizar el constructo de empatía hacia la pareja romántica y lograr con ello contar con un instrumento con características psicométricas apropiadas. Los resultados mostraron que dicho constructo -en el contexto de pareja- se compone de cuatro dimensiones: Toma de perspectiva, Empatía cognoscitiva de las emociones, Perturbación propia y Compasión empática.

La dimensión de Toma de perspectiva quedó integrada por reactivos que hacen referencia a la habilidad que posee un individuo para percibir los estados emocionales de su pareja, así como a la capacidad imaginativa para ponerse en los zapatos de su pareja o para ver las cosas desde el punto de vista de ésta. Conceptualmente este factor une a dos conceptos que si bien han sido definidos en general como empatía, son fenómenos relacionados más no elementos o aspectos o facetas a la que esta se refiere. Así, el acto de imaginar cómo uno pensaría o sentiría en la situación de otra persona (ponerse en sus zapatos) (Smith, 1759, 1853) es retomado por Mead (1934) como un juego de roles, y por Povinelli (1993) como empatía cognoscitiva e incluso por Piaget (1953) como toma de perspectiva o decentering (habilidad de considerar múltiples aspectos de una situación). Adoptar una perspectiva del yo imaginado (Stotland, 1969) es de alguna forma semejante al acto de proyectar el yo en una situación que involucra a otro (visión estética), sin embargo, el concepto en cuestión surge en un contexto interpersonal (ver Batson, 2009). En cuanto a la comparación de este factor con lo obtenido en otras medidas, se puede decir que es similar en términos de su contenido a la que Davis (1983) denominó Toma de perspectiva en la validación del IRI, a la que Díaz-Loving et al., (1986) llamaron Empatía cognoscitiva durante el proceso de validación de la EASE y a la dimensión Pensar como el paciente de la EEMJ de Hojat et al., (2002). Esta última dimensión fue encontrada al operacionalizar a la empatía en el contexto del personal médico, por tal motivo hace referencia a pensar de manera similar a los pacientes; en tanto que, los reactivos de la presente escala hacen referencia a pensar como lo hace la pareja.

De manera interesante y complementaria surge un factor que se denominó Empatía cognoscitiva de las emociones y que refleja precisamente la unión entre lo cognoscitivo y lo emocional evidente en algunas de las definiciones (e.g., Lipps, 1909; Hogan, 1969; Díaz Loving et al., 1985; Hojat et al., 2002) que justamente ven a la empatía desde la participación de la cognición en el entendimiento de las emociones que experimenta el otro, en el contexto de esta investigación, lo que experimenta la pareja. Así, este factor se define como el reconocimiento perceptual y comprehensivo que una persona tiene acerca de otra cuando esta experimenta emociones de distinta intensidad, es decir, desde algo leve hasta algo intenso e incluso manifestaciones continuas de sensibilidad emocional. Asimismo entraña tanto emociones positivas como negativas ante experiencias internas (e.g., distingo claramente cuando mi pareja se siente triste) como externas o sociales (e.g., percibo cuando alguien no le simpatiza a mi pareja). En congruencia, algunos investigadores han llamado a este conocimiento del estado interno de otra persona en sus pensamientos y sentimientos, como precisión empática (Ickes, 1993 como se cita en Batson, 2009); aunque hay que establecer que esto puede ser un poco problemático debido a que las claves emitidas por el emisor pueden ser limitadas para el agente (Kimble et al., 2002) generando cierto error en su interpretación de la realidad del otro.

En lo que respecta a la dimensión de Perturbación propia, se observa que los reactivos aquí englobados hacen referencia a los sentimientos de ansiedad, de malestar y/o de tristeza que una persona experimenta al observar que su pareja se encuentra en situaciones adversas. En términos conceptuales, 
sentirse como otra persona se siente -señala Batson (2009)- es una de las definiciones de empatía, asimismo, ha sido usada por filósofos (e.g., Darwall, 1998), neurocientíficos (Damasio, 2003) y psicólogos (Preston \& de Waal, 2002). Frecuentemente, quienes usan esta definición señalan que quien empatiza no necesita sentir exactamente la misma emoción que la otra persona, sino únicamente una parecida. La clave del uso de este término no es solo que la emoción coincida con la experimentada por la otra persona, sino la captación de esta (Hatfield, Cacioppo, \& Rapson, 1994). Saber lo que una persona puede sentir lo que otro siente, hace referencia al concepto de Levenson y Ruef (1992) denominado fisiología compartida e incluso con el contagio emocional de Hatfield et al., (1994). En el escenario de las medidas, este factor es congruente en términos conceptuales a la de Malestar personal, obtenida por Davis (1983) en el IRI y a la dimensión obtenida en el EASE de Díaz-Loving et al., (1986) que también lleva por nombre Perturbación propia.

Finalmente, el factor denominado Compasión empática agrupó una serie de reactivos que hacen referencia a la sensibilidad y ternura que se experimenta con respecto a la pareja, lo que va guiando a cierta preocupación por su estado emocional lo cual eventualmente puede llevar a tratar de ayudarla cuando lo necesite (Smith, 1759; 1853). En congruencia Batson $(1991 ; 2009)$ indica que a la compasión empática también se le conoce como preocupación empática o cuidado compasivo que involucra un toque conductual. Este concepto se refiere a la respuesta emocional generada por otra persona y a la congruencia con el bienestar percibido en esa persona. La orientación aquí se refiere al foco de la emoción, es decir, la emoción es sentida por el otro. La congruencia se refiere a la valencia de la emoción -positiva cuando el bienestar percibido es positivo, negativa cuando el bienestar es negativo-. Al hablar de congruencia se implica que el contenido de la emoción pueda ser parecido o algo semejante. Cabe indicar que este proceso también ha sido llamado Preocupación empática que obtuvo Davis (1983) en el IRI y corresponde al de Compasión empática que se encontraron en la EASE Díaz-Loving et al., (1986).
La presente investigación permite -en este punto- el análisis de dos aspectos centrales al constructo en cuestión: la empatía. En primer lugar, en lo hasta aquí expuesto permite reconocer la complejidad que posee esta variable pues las definiciones cobran matices cognoscitivos, emocionales, de distintos niveles de involucramiento o incluso traslapes únicos en la emoción propia y del otro que dan pie a que la etiquetación de los factores pueda ser múltiple en acepciones. Por lo tanto es interesante notar que la empatía está simbolizando un conglomerado de aspectos/conceptos que si bien se reconocen como diferentes, dan respuesta a la pregunta acerca de icómo se pueden saber los pensamientos y sentimientos de otra persona? (Batson, 2009). En segundo lugar, el diseño y validación de una medida como la presentada aquí, representa una contribución metodológica que da cuenta del papel de la empatía en el contexto de pareja, y que se compone tanto de elementos cognoscitivos (Toma de Perspectiva y Empatía Cognoscitiva de las Emociones) como emocionales (Perturbación propia y Cuidado compasivo). Hallazgo que es congruente con lo planteado por algunos teóricos (e.g., Eisenberg, 2000; Feshbach, N. D., \& Feshbach, S., 2011; Nickerson et al., 2011) y confirmado por otros al operacionalizar dicho constructo a través de instrumentos de autoinforme (e.g., Davis, 1983; Díaz-Loving et al., 1986; Hojat et al., 2002).

Por otra parte, el orden en el que aparecieron los factores (primero las dimensiones correspondientes al dominio cognoscitivo de la empatía y después las correspondientes al dominio emocional) sugiere que, como señalan algunos autores (e.g., Díaz-Loving et al., 1985; Eisenberg, 2000) el primer contacto hacia la realidad del otro es la toma de perspectiva, el segundo paso en este proceso de inmersión es la empatía cognoscitiva necesaria para la comprensión del estado emocional o de la situación de este(a) y posteriormente la generación de la respuesta emocional tácita y compasiva ante lo que la otra persona está sintiendo.

Desde la perspectiva de las neurociencias, los resultados aquí obtenidos son enriquecidos al considerar las contribuciones de autores como Singer y Lamm (2009) quienes indican la existencia de un 
proceso automático que surge durante la interacción y en el que se activan una serie de estructuras neuronales durante el compartir emocional, no obstante este proceso natural en los seres humanos, estos autores mencionan que la empatía es muy flexible y que las respuestas vicarias son maleables con respecto a aspectos como el contexto en el que se aprecia la emoción del otro, la relación interpersonal entre las personas o incluso la perspectiva adoptada durante la observación del otro.

Dichos hallazgos cobran relevancia si se considera que en la investigación sobre empatía en el contexto romántico, se informa que la capacidad para entender a la pareja y ponerse en su lugar, favorecen la interacción a nivel emocional entre sus miembros (Carrillo, 1999), así como el uso de un estilo de negociación integrativo ante el conflicto en el que se consideran tanto los propios intereses como los de la pareja (Aguilera, 2009). En congruencia, el estudio sobre las estrategias de co-regulación emocional (Butler \& Randall, 2013) señala cómo en la pareja se crea una sincronicidad emocional que se observa claramente en el contacto visual, la expresión facial, la prosodia, el ritmo en el discurso, la atención y fisiología automática, como el latido del corazón.

Además de lo anterior, es importante señalar que el instrumento obtenido en el presente estudio cuenta con características psicométricas adecuadas, que permiten su aplicación en muestras similares a la aquí empleada para evaluar la empatía específicamente en el contexto de pareja. Adicionalmente, dicho instrumento presenta una operacionalización de la empatía hacia la pareja culturalmente relevante, pues fue validado en una muestra de mexicanos de la Ciudad de México.

\section{Referencias}

Aguilera, M. I. (2009). Comprensión Empática y Estilos de Negociación en la relación de pareja: Herramientas de mediación. Revista Internacional de Psicología, 10(2), 1-14.

Batson, C. D. (1991). The Altruism question: toward a social-psychological answer. Hillsdale, NJ: Erlbaum.

Batson, C. D. (2009). These things called empathy: eight related but distinct phenomena. En J. Decety and
W. Ickes (Eds.), The Social Neuroscience of Empathy (pp. 3-15). Cambridge, Mass: A Bradford Book, The MIT Press.

Bernhardt, B. C., \& Singer, T. (2012). The neural basis of empathy. Annual Review of Neurosciences, 35, 1-23.

Blázquez-Alonso, M., Moreno-Manso, J. M., \& GarcíaBaamonde Sánchez, M. E. (2009). Inteligencia emocional como alternativa para la prevención del maltrato psicológico en la pareja. Anales de Psicología, 25(2), 250-260.

Butler, E., \& Randall, A. (2013). Emotional Co-regulation in Close Relationships. Emotion Review, 5(2), 202-210.

Carlson, J. G., \& Hatfield, E. (1992). Psychology of Emotion. USA: Harcourt Brace Jovanovich College Publishers.

Carrillo, J. (1999). Evaluación del nivel empático en parejas primigestas antes y después del parto. (Tesis inédita de maestría). Instituto Mexicano de la Pareja, México.

Damasio, A. R. (2003). Looking of Spinoza: Joy, sorrow and the feeling brain. Orlando, FL: Harcourt.

Darwall, S. (1998). Empathy, sympathy, care. Philosophical Studies, 89, 261-282.

Davis, M. H. (1983). Measuring individual differences in empathy: Evidence for a multidimensional approach. Journal of Personality and Social Psychology, 44, 113-126.

Decety, J., \& Jackson, P. L. (2004).The functional architecture of human empathy. Behavioral and Cognitive Neuroscience Reviews, 3, 71-100.

Díaz-Loving, R., Andrade-Palos, P., \& Nadelsticher-Mitrani, A. (1986). Desarrollo de la Escala Multidimensional de Empatía (EASE). Revista de Psicología Social y Personalidad, 2(1), 3-11.

Díaz-Loving, R., González-Varela, M., La Rosa, J., \& Nina-Estrella, R. (1985). Empatía: Antecedentes históricos y su relación con conductas prosociales y antisociales. Revista de Psicología Social y Personalidad, 1(2), 77-92.

Eisenberg, N. (2000). Emotion, regulation, and moral development. Annual Review of Psychology, 51, 665-697.

Fernández-Pinto, I., López-Pérez, B., \& Márquez, M. (2008). Empatía: Medidas, teorías y aplicaciones en revisión. Anales de Psicología, 24(2), 284-298. 
Feshbach, N. D., \& Feshbach, S. (2011). Empathy and Education. En J. Decety, \& W. Ickes (Eds.), The Social Neuroscience of Empathy (p.p. 85-97). Massachusetts: The MIT Press.

González-Ortega, I., Echeburúa, E., \& Corral, P. (2008). Variables significativas en las relaciones violentas en parejas jóvenes: Una revisión. Psicología Conductual, 16(2), 207-225.

Gordon, R. M. (1995). Sympathy, simulation and the impartial spectator. Ethics, 105, 727-742.

Guerrero, L. K., Andersen, P. A., \& Afifi, W.A. (2011). Communicating closeness: affection, immediacy, and Social Support. En L. Guerrero, P. Andersen $\&$ W. Afifi. (Eds.). Close encounters communication in relationships (pp. 125-150). California: Sage

Hatfield, E., Cacioppo, J. T., \& Rapson, R. L. (1994). Emotional contagion. New York: Cambridge University Press.

Hatfield, E., Rapson, R. L., \& Le Y. Ch., L. (2009). Emotional contagion and empathy. En J. Decety and W. Ickes (Eds.), The Social Neuroscience of Empathy (pp. 19-30). Cambridge, Mass: A Bradford Book, The MIT Press.

Hoffman, M. L. (2000). Empathy and moral development: implications for caring and justice. New York: Cambridge University Press.

Hogan, R. (1969). Development of an Empathy Scale. Counsulting and Clinical Psychology, 33, 307-316.

Hojat, M., Gonnella, J. S., Nasca, T. J., Mangione, S., Vergare, M., \& Magge, M. (2002). Physician Empathy: Definition, Components, Measurement, and Relationship to Gender and Specialty. American Journal of Psychiatry, 159, 1563-1569.

Kerlinger, F. N., \& Lee, H. B. (2002). Investigación del Comportamiento. México: McGraw-Hill

Khöler, W. (1947). Gestalt psychology. New York: The New American Library.

Kimble, C., Hirt, E., Díaz Loving, R., Hosch, H. Lucker, G. W., \& Zárate, M. (2002). Psicología Social de las Américas. México: Prentice Hall.

Laurenceau, J. P., \& Kleinman, B. M. (2006). Intimacy in Personal Relationships. En A.L. Vangelisti \& D. Perlman (Eds.). The Cambridge Handbook of Personal Relationships (pp. 637-656). New York: Cambridge University Press.
Levenson, R. W., \& Ruef, A. M. (1992). Empathy: A physiological substrate. Journal of Personality and Social Psychology, 63, 234-246.

Lipps, T. (1909). Leitfaden der Psychologie. Leipzig: Wilhelm Engelmann Verlag.

Mead, G. H. (1934). Mind, self, and society. Chicago: University of Chicago Press.

Mehrabian, A., \& Epstein, N. (1972). A measure of emotional empathy. Journal of Personality, 40, 525-543.

Nedelsticher-Mitrani, A., Díaz-Loving, R., \& NinaEstrella, R. (1983). La empatía: iUnidimensional o multidimensional? Enseñanza e Investigación en Psicología, IX(2), 247-254.

Nickerson, R. S., Butler, S. F., \& Carlin, M. (2011). Empathy and Knowledge Projection. En J. Decety \& W. Ickes (Eds.), The Social Neuroscience of Empathy (p.p. 43-56). Massachusetts: The MIT Press.

Pérez-Albéniz, A., De Paúl, J., Etxeberría, J., Montes, M. P., \& Torres, E. (2003). Adaptación de Interpersonal Reactivity Index (IRI) al español. Psicothema, 15(2), 267-262.

Perlman, D. (2000). El lado oscuro de las relaciones. Revista de Psicología Social y Personalidad, XVI(2), 95-121.

Piaget, J. (1953). The origins of intelligence in the child. New York: International Universities Press.

Povinelli, D. J. (1993). Reconstructing the evolution of mind. American Psychologist, 48, 493-509.

Preston, S. D., \& de Waal, F. B. M. (2002). Empathy: its ultimate and proximate bases. Behavioral and Brain Sciences, 25, 1-72.

Reyes-Lagunes, I.,\& García y Barragán, L. F. (2008). Hacia un procedimiento de validación psicométrica culturalmente relevante. En S. Rivera-Aragón, R. Díaz-Loving, R. Sánchez-Aragón y I. ReyesLagunes (Eds.), La Psicología Social en México XII (pp. 625-630). México: AMEPSO.

Singer, T., \& Lamm, C. (2009). The Social Neuroscience of Empathy. Annals of the New York Academy of Sciences, 1156, 81-96.

Smith, A. (1759; 1853). The theory of moral sentiments. London: Alex Murray.

Stotland, E. (1969). Exploratory investigation of empathy. En L. Berkowitz (Ed.), Advances in Experimental Social Psychology (Vol. 4 pp. 271-313). New York: Academic Press. 